\title{
Aplicación de MÉTODOS MATEMÁTICOS PARA EL ANÁLISIS DEL DISEÑO DE PROYECTOS ARQUUTECTÓNICOS
}

\author{
Application of mathematical methods for the analysis of the design of architectural projects
}

\begin{tabular}{|c|c|}
\hline \multicolumn{2}{|c|}{$\begin{array}{c}\text { Jonathan Hernández-Omaña* ERle García-Estrada** } \\
\text { David liarra-González }{ }^{* * *}\end{array}$} \\
\hline & $\begin{array}{l}\text { Fecha de recibido: } \\
\text { 30 Abril } 2020 \\
\text { Fecha de aceptado: } \\
4 \text { Agosto } 2020\end{array}$ \\
\hline & $\begin{array}{l}\text { *Instituto Universitario del Estado de México, } \\
\text { México. } \\
\text { jho_20oooo@@hotmail.com }\end{array}$ \\
\hline & $\begin{array}{l}\text { **Universidad Autónoma del Estado de México, } \\
\text { México. } \\
\text { erlemanzana@gmail.com }\end{array}$ \\
\hline & $\begin{array}{l}\text { ***Universidad Autónoma del Estado de México, } \\
\text { México. } \\
\text { ibarradav@gmail.com }\end{array}$ \\
\hline
\end{tabular}

RESUMEN. El presente artículo muestra la aplicación de planteamientos matemáticos para la toma de decisión aplicados a etapas iniciales del diseño de proyectos arquitectónicos, particularmente esta metodología puede ser empleada para analizar la distribución de espacios en las etapas tempranas de diseño.

Este estudio se fundamenta en el análisis y la administración de características del programa arquitectónico a través de la implementación demetodologías detoma de decisión multicriterio (мСDM), las cuales, si bien no resultaran en observaciones objetivas, permitirán la administración y entendimiento de las necesidades, actividades y espacios; los hallazgos del presente trabajo permitirán tomar decisiones referentes a la distribución de espacios y recursos, basando su elección en la identificación numérica de juicios e incluso la creación de reflexiones combinadas que conjunten las observaciones realizadas por el diseñador y los usuarios.

Los resultados de esta propuesta no solo permiten la determinación de una distribución espacial ordenaday explicita, sinotambién la posible mejora y combinación de juicios, el análisis de comportamientos, y la posibilidad de considerar nuevas técnicas de distribución y conceptualización arquitectónica, por lo cual es importante mencionar que el objetivo del presente trabajo no es evaluar los juicios, sino proponer un análisis matemático de una problemática y su traducción a una expresión gráfica de la manera en la que se abordará la distribución de un programa arquitectónico.

Palabras clave: diseño arquitectónico, modelos matemáticos, sistemas alternativos de toma de decisiones.
ABSTRACT. This article shows the application of mathematical approaches for decision-making applied to initial stages of architectural project design, particularly this methodology can be used to analyze the distribution of spaces in the early stages of design.

This study is based on the analysis and administration of characteristics of the architectural program through the implementation of multi-criteria decisionmaking methodologies (мСDM) which, although they will not result in objective observations, will allow the administration and understanding of the needs, activities and spaces; The findings of the present work will allow making decisions regarding the distribution of spaces and resources, basing their choice on the numerical identification of judgments and even the creation of combined reflections that combine the observations made by the designer and the users.

The results of this proposal allow not only the determination of an ordered and explicit spatial distribution, but also the possible improvement and combination of judgments, the analysis of behaviors, and the possibility of considering new distribution techniques and architectural conceptualization, for which it is It is important to mention that the objective of this work is not to evaluate the judgments, but to propose a mathematical analysis of a problem and its translation into a graphic expression of the way in which the distribution of an architectural program is approached.

Key words: architectural design, mathematical models, alternative decision-making systems. 
$\mathrm{E}$

1 presente trabajo pretende aproximar a los diseñadores a metodologías matemáticas para el diseño y distribución de programas arquitectónicos, lo que permitirá ofrecer más herramientas de aproximación a la gestión y distribución de espacios basados en las necesidades sentidas de los usuarios, las cuales pueden ser difíciles de expresar al realizar los procesos analíticos de diseño, ya que como será abordado a continuación, los procesos analíticos para determinar suelen no contemplar de manera específica métodos cuantitativos particulares que permitan expresar una conclusión particular y trasladarla a una representación o distribución gráfica del mismo.

En lo referente al proceso analítico del diseño arquitectónico, T. White (1987) señala que por tradición supedita los conceptos de espacio a un análisis de necesidades, requerimientos o referencias, las cuales contrasta con experiencias y conocimientos de las que crea una imagen mental general, que pretende pasar de las necesidades del proyecto a una solución material (o edificación), dicho autor agrupa las cuestiones de composición y magnitud, conceptos que posteriormente se integran en la segunda etapa de su metodología denominada "Diseño esquemático", donde se abordan las temáticas de agrupación y zonificación del programa arquitectónico (figura 1), sin embargo, no presenta ninguna metodología de aproximación específica para este enfoque.

\section{$+$}

señala que el análisis para determinar un espacio comprenderá las fuerzas, el genius loci, la naturaleza y el arte, la poesía, el significado en el uso, los materiales y el significado, pero no presenta un método para realizar este proceso, como se muestra en la figura 2.

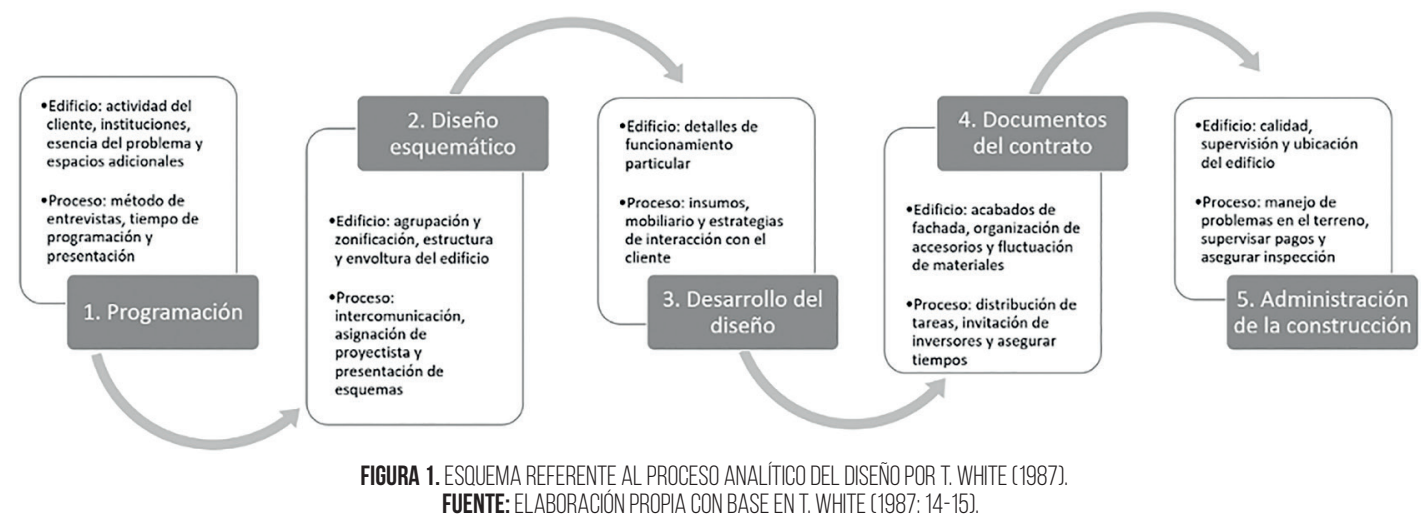




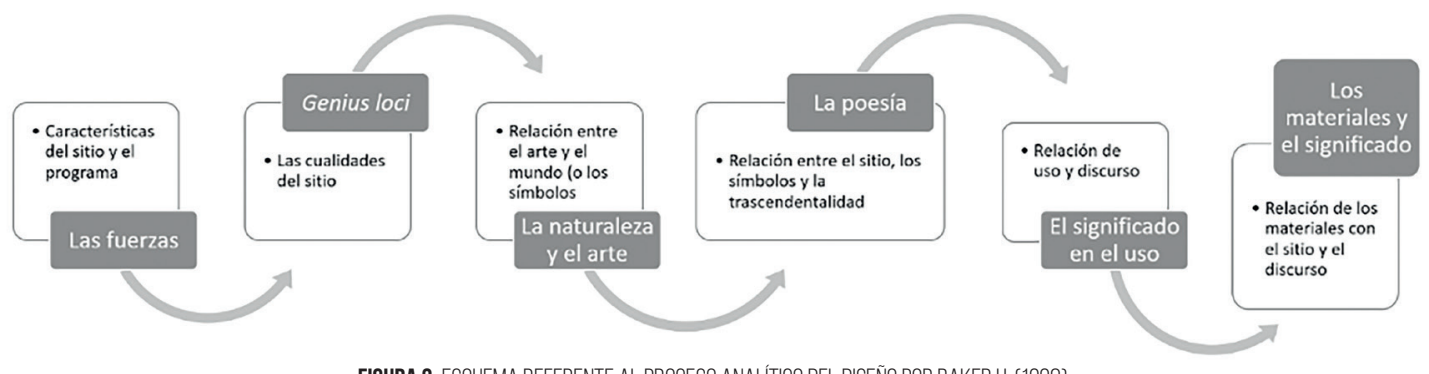

FIGURA 2. ESQUEMA REFERENTE AL PROCESO ANA LítICO DEL DISEÑO POR BAKER H. (1998). FUENTE: ELABORACIÓN PROPIA CON BASE EN BAKER H. (1998).

Finalmente, con respecto al estado del arte en los métodos para el proceso del diseño arquitectónico, Szilágyi \& Bakai (2019) presentan una investigación que identifica los retos de los estudiantes de Arquitectura hacen frente al diseñar, en este análisis se pretende determinar la manera en que las metodologías de diseño actuales han impactado a la eficiencia de diseño, tanto en actividades escolares como laborales; producto de su análisis identificaron tres etapas y problemáticas: concepción, elaboración y propuesta, en la primera uno de los elementos que presenta mayor dificultad para los estudiantes es la modelización del sitio y el entorno, en la segunda la pérdida de tiempo en la traducción gráfica del diseño y la carencia de conocimiento en el software para representar, finalmente, en la tercera etapa la demanda de automatización existe pérdida de información debido a las técnicas de trabajo inapropiadas o poco eficientes.

En lo particular, se identificó que los alumnos podrían obtener una experiencia más eficiente si sus diseños fueran determinados durante el proceso de trabajo, para conocer cómo abordar cada parte del proyecto y las herramientas que estos requieren, lo cual a su vez les permitirá establecer expectativas con respecto a cada área y parte del proceso de diseño; es decir, ¿qué tienen que obtener de cada parte de su planteamiento metodológico?

Por ello el presente trabajo pretende realizar una aproximación a la aplicación de una metodología MCDM para la propuesta de una distribución espacial y no la evaluación de los juicios emitidos, en donde se realice una aproximación comparativa de las expectativas y pensamientos de grupos de análisis particulares, siendo en este caso un proyecto existente, usuarios sin experiencia en metodologías de distribución y arquitectos con experiencia práctica en la realización de los mismos, se decidió emplear una aproximación de este tipo debido a como lo presenta Harputlugil (2018), las metodologías de este tipo permiten analizar información obtenida a través de encuestas y entrevistas, puede ser aproximada de forma que se obtenga resultados numérico que sean comparables, medibles y consistentes (aunque el autor determina que existen límites en el tipo de comparaciones que se realizan dependiendo del tipo de proyecto).

Esta aproximación también ha sido analizada por Elango \& Devadas (2014), quienes realizaron un ejercicio de evaluación multicriterio de las propuestas de un proyecto tipo de un grupo de alumnos en los cuales fue posible identificar que el juicio intuitivo $\mathrm{y}$ las decisiones realizadas en un solo objetivo no pueden ser usadas para predecir completamente la mejor solución, y que la mayoría de la información importante que puede mejorar la calidad del diseño se realiza en las etapas del pre-diseño, señalando que existe un mayor beneficio en la incorporación de datos y metodologías adicionales en estas etapas del diseño.

Una vez examinadas de manera general los métodos planteados por diversos autores, así como la caracterización de las carencias y posibilidades de mejora de la metodología de diseño, el presentetrabajo pretendevislumbrar una aproximación cuantitativa para las etapas tempranas del diseño arquitectónico mediante el uso de técnicas particulares que permitan a los arquitectos y estudiantes identificar de manera cuantitativa la problemática y escala de las propuestas que estos realizan.

El presente trabajo pretende realizar una aproximación a una aplicación particular de metodología de diseño arquitectónico mediante el uso de un modelo matemático, donde se propone como punto de partida para la propuesta de partido arquitectónico 
básico en función a una aproximación numérica; a su vez es necesario destacar que esta aplicación se realiza en las etapas tempranas y conceptuales o analíticas del proyecto, por lo tanto no se encuentra relacionado con aplicaciones de tipo BIM (de gestión, modelado o administración).

\section{PROCESO DE ANÁLISIS JERÁRQUICO [AHP]}

El proceso de decisión o elección en etapas tempranas del diseño es una temática muy compleja al presentar al individuo la necesidad de procesar elementos intangibles y traducirlos a una expresión material. Mu \& Pereyra-Rojas (2017) señalan que si bien se da por hecho que los individuos son capaces de procesar de manera racional la información y que en muchos casos pretenden tomar decisiones que los lleven a maximizar los beneficios de sus decisiones, estos pueden ser fácilmente influenciados por sus prejuicios cognitivos, la manera de ordenar la información, o el desconocimiento de procesos de sistematización y análisis de los datos; por lo cual se propone emplear una metodología que permita identificar de manera numérica las observaciones realizadas por cada uno de los individuos y analizar cuáles son las posibles influencias que modifican las posturas de los mismos, por ello se propone aplicar una metodología cuantitativa de análisis espacial en un caso específico, siendo la elegida, en este caso, el proceso de análisis jerárquico (AHP, por sus siglas en inglés).

El AHP es parte de las técnicas para la toma de decisión multicriterio (Multicriteria Decision-making o MCDM), la cual autores como Aragonés et al., (2014) señalan que es parte de las técnicas desarrolladas para ayudar a los tomadores de decisiones a orientar la elección de decisiones en problemas complejos de manera sistemática

\section{Para realizar el análisis AHP \\ ES NECESARIO ESTRUCTURAR EL PROBLEMA EN UNA JERARQUIA Y HACER UNA SERIE DE MEDIDAS RELATIVAS MEDIANTE LA ESCALA PROPUESTA POR THOMAS SAATY, QUE ES LA "ESCALA FUNDAMENTAL DE SAATY"}

y ordenada, al emplear de este tipo de técnica para desarrollar un partido arquitectónico particular permitirá identificar de manera cuantitativa la forma en la que los decisores dimensionan los espacios basados en su conocimiento, expectativas y necesidades.

Para realizar el análisis AHP es necesario estructurar el problema en una jerarquía y hacer una serie de medidas relativas mediante la escala propuesta por Thomas Saaty, que es la "escala fundamental de Saaty" (figura 3).

Mediante esta escala el decisor determinará la prioridad de un espacio con respecto a otro (creando comparaciones, las cuales usualmente no suelen ser consideradas en una determinación general), siendo en este caso el criterio de primer nivel 1 (de igual importancia) y las prioridades de cada uno de los criterios de segundo nivel establecidos por los individuos encuestados.

El proceso de análisis usualmente consta de cinco pasos hasta llegar a la elección de una alternativa, sin embargo, para el presente trabajo exploratorio únicamente se llegará a identificar las prioridades e inconsistencia

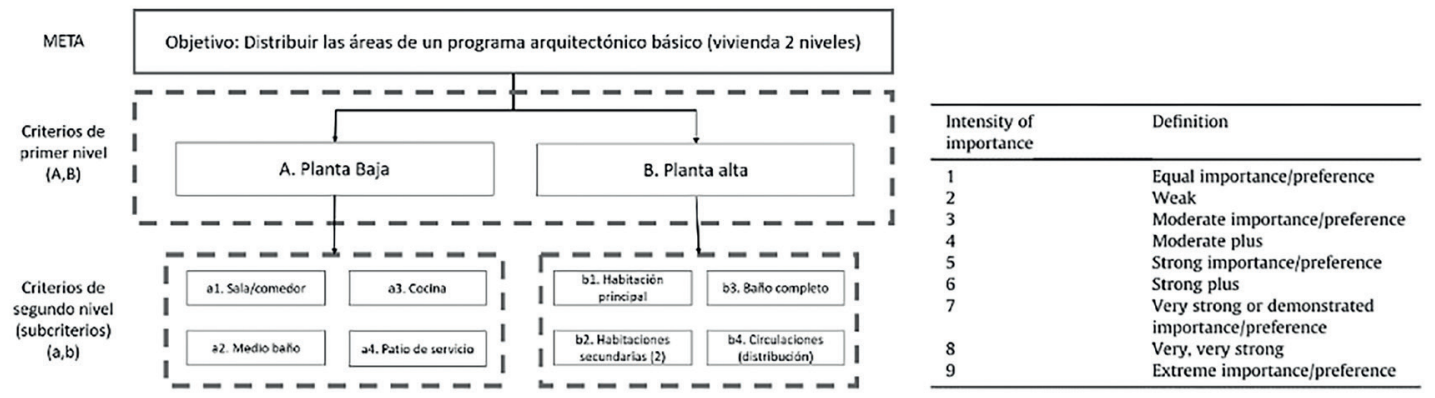

FIGURA 3. PROBLEMA EXPRESADO DE MANERA JERÁROUUICA Y ESCALA FUNDAMENTAL DE SAATY. FUENTE: INFORMACIÓN PROPIA CON BASE EN ARAGONÉS ET AL. (2014: 224). 
de los juicios emitidos, ya que en este caso en particular no se pretende llegar a la elección de la postura de un individuo (debido a que cada uno presenta una determinación), si no explorar los juicios expresados por diferentes personas para encaminar el proceso en etapas tempranas del proceso de diseño y determinar si es posible realizar aproximaciones matemáticas de una problemática de tipo subjetiva:

1. Se estructura el problema como una jerarquía y se definen niveles de análisis, siendo el elemento principal la meta del estudio y los niveles consecuentes serán los criterios tangibles o intangibles a analizar; para este caso el último nivel de alternativas no será desarrollado, como se mostró en la figura 3.

2. Se identifican las prioridades de cada criterio (esto es obtenido al comparar los elementos de un mismo nivel con la escala fundamental de Saaty en una matriz pareada A que es obtenida de los juicios emitidos por el decisor mediante una serie de preguntas comparativas y procesadas en el software Super Decisions, como se muestra en la figura 4.

Posteriormente, se determinará el ratio de consistencia para identificar el índice de consistencia de los juicios emitidos con $\mathrm{CR}=\mathrm{CI} / \mathrm{RI}$, donde $\mathrm{CI}=(\lambda \max -\mathrm{n}) /(\mathrm{n}-1) \mathrm{y}$ $\lambda$ max es el máximo eigenvalor de la matriz A, usualmente este CI determina si las observaciones pueden ser tomadas como parte de la decisión si éstas son menores al valor máximo determinado (0.05, 0.08 o 0.10 en el caso de que n sea más de 5), en el caso de que el juicio supere el límite del máximo de inconsistencia éste deberá ser revisado, ya que, puede que el decisor no comprenda la escala, el problema o el impacto de cada juicio emitido, sin embargo, para dicha aplicación se procederá con el cI que presenten, aun si éste excede el límite de inconsistencia al ser un ejercicio exploratorio.

Una vez identificado el CI y aceptado el juicio se procede a determinar las prioridades del vector o cada criterio, que son obtenidas de la matriz pareada A, las cuales son calculadas con el correcto eigenvector de la matriz pareada A, permitiendo identificar las prioridades locales y globales (criterios y subcriterios).

Finalmente, serán sintetizadas las prioridades locales con todos los criterios globales (o normalizadas), donde la prioridad global será 1 y la suma de todas las prioridades locales o secundarias sumará ese mismo 1 (es decir, explicando cómo todas las prioridades conforman la totalidad del juicio o el 100\% repartido en la aplicación porcentual).

Esta aplicación permitirá identificar la manera en la que los decisores formaron juicios con respecto a una problemática compleja como es la distribución de espacios y jerarquizar la distribución de un programa arquitectónico mediante un procedimiento sistemático, que permite dividir la aproximación en subproblemas e

$$
\mathrm{A}=\left[\begin{array}{cccc}
1 & a_{12} & \cdots & a_{1 n} \\
a_{21} & 1 & \cdots & a_{2 n} \\
\vdots & & \ddots & \vdots \\
a_{n 1} & a_{n 2} & \cdots & 1
\end{array}\right], \text { where } a_{j i}=1 / a_{i j} i, j=1, \ldots, n
$$
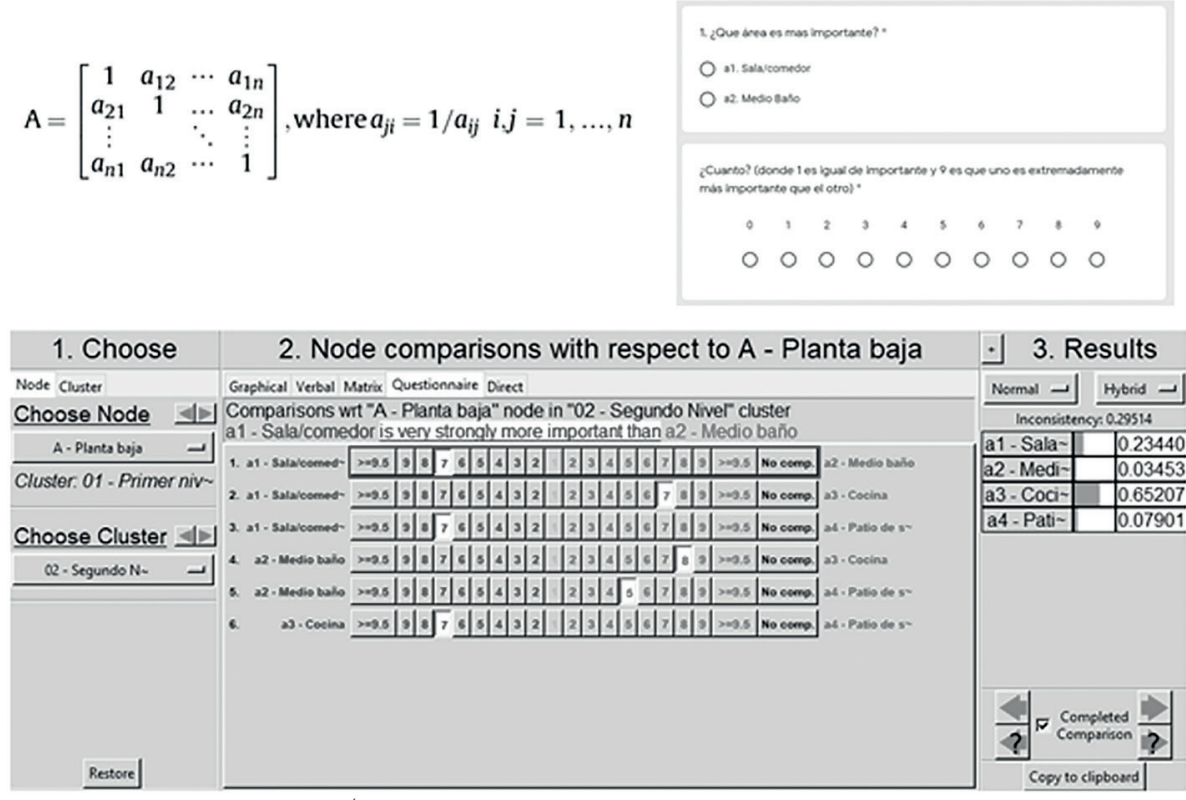

FIGURA 4. REPRESENTACIÓN DE LA MATRIZ A Y CAPTURA DE DATOS REALIZADA EN EL SOFTWARE SUPER DECISIONS FUENTE: ARAGONÉS ET AL. (2014: 224) Y SUPER DECISIONS. 
identificar la importancia individual de cada una, ya que como será contrastado con la distribución porcentual directa ésta puede no estar relacionada de manera directa.

En segundo lugar, identificar los comportamientos particulares o identificación de consistencia de ambas distribuciones, ya que en el caso de la distribución porcentual se hace un énfasis significativo únicamente en una distribución conceptual simple, es decir, la distribución de un total de espacio; sin embargo, en esta aproximación los decisores no suelen identificar la importancia cualitativa de los espacios; mientras que en la aplicación del cuestionario de prioridades AHP se les preguntará cualitativamente sobre la importancia de cada espacio con respecto a otros de manera individual a diferencia de la aproximación generalizada del enfoque porcentual (cuantitativo).

Para ello los juicios expresados por las personas encuestadas serán vaciadas en el software Super Desicions de dos maneras, una será llenando los datos referentes al porcentaje expresado (lo cual supone una limitación en la inconsistencia de sus juicios al contemplar a la problemática como un elemento delimitado por un porcentaje máximo (100\%) y llenado como porcentaje de distribución y otra mediante preguntas comparativas, donde los encuestados probablemente presenten juicios más inconsistentes al no tener esta limitación cualitativa del problema, ya que rara vez se analiza una problemática considerando en todo momento la totalidad de la problemática y suelen enfocarse únicamente en las preguntas puntuales (es decir, se desglosa la problemática, pero no se realizan consideraciones posteriores de integración), como se muestra en la figura 5 .

Finalmente, se contrastarán los resultados mediante un análisis comparativo entre las distribuciones porcentuales y los índices de inconsistencia expresados en ambas distribuciones (cuantitativa y cualitativa).

\section{LIMITANTES DEL ANÁLISIS}

Las limitantes y restricciones del análisis a realizar debido a su naturaleza mixta son las siguientes:

-El presente estudio tiene una naturaleza exploratoria en principio y experimental en menor medida.

- La comparación y contraste de datos expresados por arquitectos-usuarios y la distribución porcentual de prioridades es limitada al no contar con un instrumento exploratorio más completo (instrumento

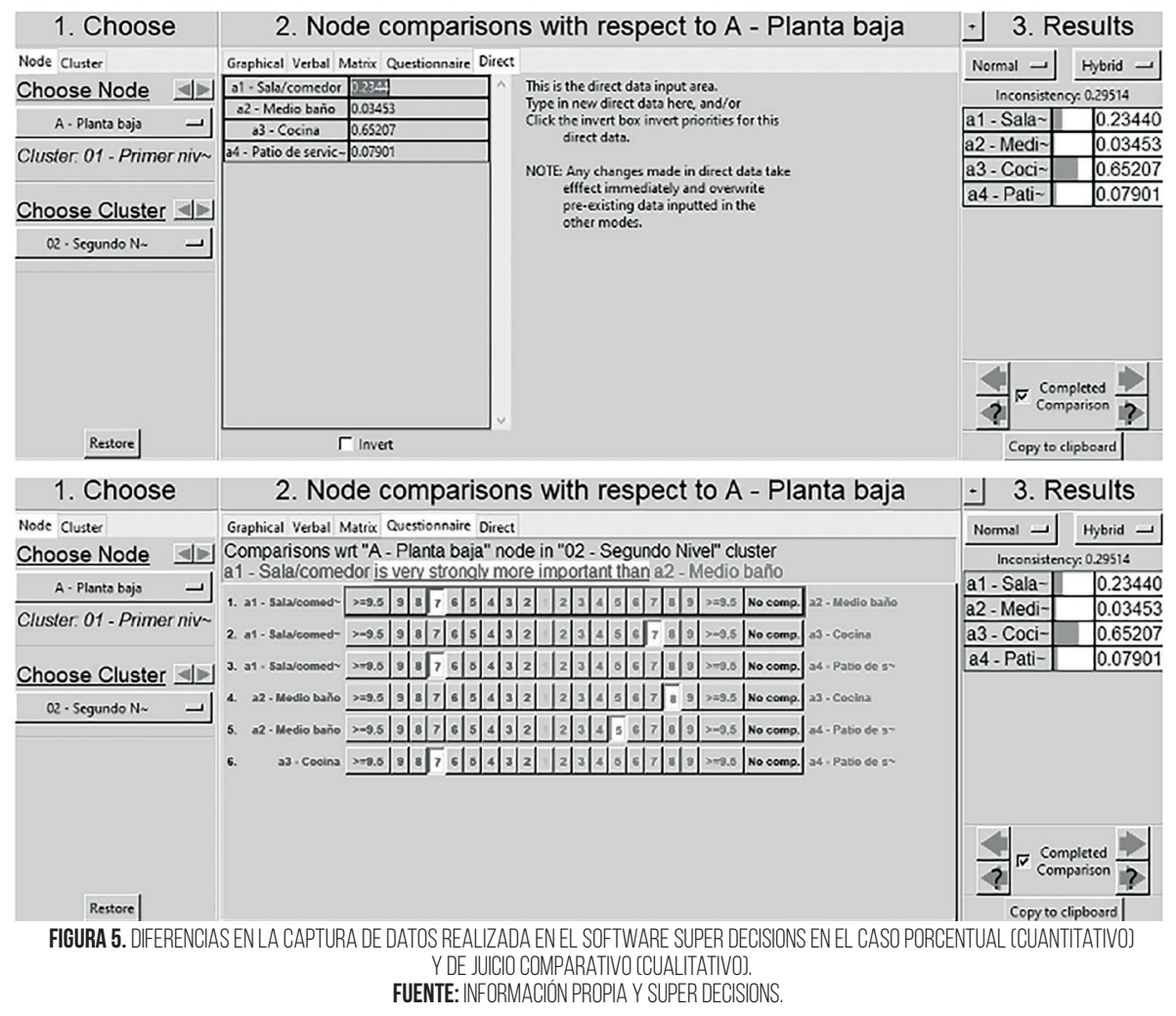


de entrevista más específico), por lo cual las observaciones a realizar serán generales e incluso anecdóticas al contar con una muestra tan limitada), sin embargo, esta limitante puede ser superada al emplear muestreos más amplios en persona.

-Los juicios expresados, tanto por los arquitectos como usuarios, son expresiones subjetivas, por lo cual, es posible que ambas muestras tengan lagunas con respecto a la distribución del planteamiento. El índice de inconsistencia en este caso estará limitado por las estrategias, si se adjuntara al planteamiento la cantidad de metros cuadrados, distribución porcentual y prioridad existirían cambios en los juicios expresados al tener una imagen numérica tangible, por ejemplo: considerar que si se destina un $16 \%$ de área a un medio baño si el planteamiento por planta es de 100 $\mathrm{m}^{2}$ dicha distribución corresponde a $16 \mathrm{~m}^{2}$ $\left(4 \times 4 \mathrm{~m}^{2}\right)$.

-El conocimiento ergonómico de mobiliario específico requerido con el que relacionan su planteamiento de distribución.

Una vez identificadas de manera general las limitantes y restricciones del análisis se procederá con una breve introducción a la metodología a emplear para realizar un análisis cualitativo-cuantitativo de las expresiones emitidas por los miembros de la muestra.

\section{METODOLOGÍA}

La metodología a desarrollar para la presente propuesta incorpora tres etapas en las cuales se realizará:

- Obtención de datos a través de un instrumento en línea.

-Análisis de juicios, alternativas y resolución de posibilidades.
- Generación de un diagrama o modelo de acción para la problemática específica.

Para realizar dichos trabajos serán empleados los métodos de encuesta, donde será presentada una problemática, se analizarán los juicios expresados mediante el proceso de análisis jerárquico, y finalmente, se realizará la interpretación de resultados en un diagrama de prioridades y partido general de la propuesta.

En primer lugar, se establecerá un reto de propuesta de distribución de proyecto arquitectónico base de una casa habitación de interés medio de dos plantas que cuenta con un programa arquitectónico de aproximadamente $145 \mathrm{~m}^{2}$, el cual presenta la distribución de espacios aproximada de la tabla 1.

Para trasladar esta problemática particular al campo del diseño se considerara el objetivo como la propuesta de distribución y administración del espacio inherente a este tipo de programa arquitectónico, según los juicios de cada uno de los encuestados, los criterios en este caso serán los componentes del programa arquitectónico; y en el caso de las alternativas éstas a nivel interno serán la jerarquía (en función a la magnitud relativa) de los espacios, es importante mencionar que las alternativas de elección en este caso podrán ser determinadas en función a diferentes criterios.

Una vez identificado un programa arquitectónico base se le hará un cuestionario a un grupo de arquitectos y usuarios, dicho instrumento consta de dos secciones, inicialmente se les pedirá determinar una distribución en función a un porcentaje de espacio que cada uno destinaría a cada uno de los componentes en cada planta, y en segundo se hará un ejercicio de consulta AHP para determinar el mismo programa, pero en esta ocasión en

TABLA 1. DISTRIBUCIÓN DEL PROGRAMA ARQUUTECTÓNICO EXISTENTE APROXIMADO

\begin{tabular}{|c|c|c|c|c|c|}
\hline \multicolumn{2}{|l|}{ Terreno } & \multicolumn{2}{|c|}{ PB } & \multicolumn{2}{|l|}{ PA } \\
\hline \multicolumn{2}{|l|}{$120 \mathrm{~m}^{2}$} & \multicolumn{2}{|c|}{$72.5 \mathrm{~m}^{2}$} & \multicolumn{2}{|l|}{$72.5 \mathrm{~m}^{2}$} \\
\hline Construido & $72.5 \mathrm{~m}^{2}$ & Sala/Comedor & $45 \mathrm{~m}^{2}$ & Habitación principal (con baño) & $35 \mathrm{~m}^{2}$ \\
\hline Libre (estacionamiento y patio) & $47.5 \mathrm{~m}^{2}$ & Medio baño & $3 m^{2}$ & Habitaciones secundarias & $25 \mathrm{~m}^{2}$ \\
\hline Total & $120 \mathrm{~m}^{2}$ & Cocina & $20 \mathrm{~m}^{2}$ & Baño completo compartido & $5 \mathrm{~m}^{2}$ \\
\hline & & Patio de servicio & $4.5 \mathrm{~m}^{2}$ & Distribución & $7.5 \mathrm{~m}^{2}$ \\
\hline \multicolumn{6}{|c|}{ Porcentajes de construcción } \\
\hline Construido & $60 \%$ & Sala/Comedor & $31 \%$ & Habitación principal (con baño) & $24 \%$ \\
\hline Libre (estacionamiento y patio) & $40 \%$ & Medio baño & $2 \%$ & Habitaciones secundarias & $17 \%$ \\
\hline \multirow[t]{3}{*}{ Total } & $100 \%$ & Cocina & $14 \%$ & Baño completo compartido & $3 \%$ \\
\hline & & Patio de servicio & $3 \%$ & Distribución & $5 \%$ \\
\hline & & Total & $50 \%$ & Total & $50 \%$ \\
\hline
\end{tabular}

FUENTE: INFORMACIÓN PROPIA Y SUPER DECISIONS. 
función a las prioridades expresadas por cada individuo; una vez obtenida las muestras y realizadas las entrevistas, las respuestas expresadas por la muestra serán comparadas con el proyecto arquitectónico existente.

Los cuestionarios fueron distribuidos de manera digital (a través de google forms) y uno se realizó de manera directa para elaborar una prueba piloto del instrumento, el cual determinó que era necesario realizar breves introducciones a las escalas y temáticas a analizar, así como ofrecer un espacio en el cual los encuestados pudieran externar cualquier duda o comentario sobre el instrumento (herramienta que solo fue empleada en uno de los casos), la aplicación en persona y lo expresado por uno de los encuestados ofrece elementos de importancia que serán considerados para la extracción de datos.

En la última etapa y producto de la aplicación del instrumento fue posible obtener información cualitativa y cuantitativa respecto a la manera en la que cada uno de los encuestados realizó sus distribuciones; es posible identificar comportamientos en lo referente a la inconsistencia de los juicios expresados por cada una de las personas encuestadas mediante el uso del programa Super Decisions e identificar comportamientos de grupos (en este caso arquitectos y usuarios), y finalmente generar una aplicación gráfica para expresar los juicios expresados de manera simplificada.

\section{RESULTADOS Y DISCUSIÓN}

De la aplicación del instrumento fueron obtenidas diez muestras, la mitad correspondían a arquitectos y la otra mitad a usuarios (sin conocimientos sobre distribución de espacios), en la cuales se realizaron dos conjuntos de preguntas, uno en donde se les pidió distribuir el programa arquitectónico (criterios) en función al porcentaje de espacio (100\%) con un enfoque cuantitativo y otra donde se realizó la misma actividad, pero en función a preguntas pareadas de cada uno de los criterios con un enfoque cualitativo, donde el objetivo era la distribución, los criterios de primer nivel cada una de las plantas del proyecto y los subcriterios cada uno de los componentes del programa arquitectónico; de las muestras obtenidas en ambos grupos fueron identificados los siguientes comporta- mientos:

- En lo referente al análisis porcentual del espacio destinado a cada área del proyecto, los resultados promedio de las expresiones de los arquitectos y usuarios (figura 6), es posible identificar que existieron grandes variaciones en lo individual en ambos grupos; al integrar el porcentaje de esta muestra el índice de inconsistencia en el caso de los arquitectos es menor con respecto al proyecto existente, pero en el caso de los usuarios la inconsistencia fue superior (especialmente en el caso de los baños, habitaciones y sala/comedor).

- En lo particular se encontró una inconsistencia promedio de juicios expresados por los arquitectos de $1.39 \%$, lo cual puede ser considerado dentro del Índice de consistencia máximo (CI máx. 5\% determinado por el número de variables o n) y con una consistencia mayor a la del proyecto existente $(1.52 \%)$; mientras que en el caso de los usuarios éste tuvo un promedio de $1.77 \%$, es decir, una inconsistencia mayor que la de los juicios de los arquitectos, pero que también podría ser considerado un CI aceptable, en este caso no se realiza la elección de una alternativa, sino más bien se establece la partida de una distribución de un programa arquitectónico basado en las prioridades expresadas, por lo cual el índice de inconsistencia obtenido en ambos planteamientos por el programa Super Decisions solamente es un parámetro de medición del eigenvector de las prioridades expresadas por el individuo.

En este tipo de análisis, el índice de inconsistencia será bajo, debido a que se tiene una comprensión delimitada de la problemática, al tener que repartir un $100 \%$ de área el que no puede ser excedido; dicho de otra manera, los juicios se mantienen consistentes al limitar el espacio a distribuir.

Del análisis de los juicios emitidos por distribución porcentual es posible identificar de manera gráfica los comportamientos con respecto a la distribución delos espacios, como se muestra en la figura 7; en donde es posible identificar la diferencia en la distribución espacial de las áreas determinadas en el proyecto arquitectónico.

En este análisis fueron expresadas dos 


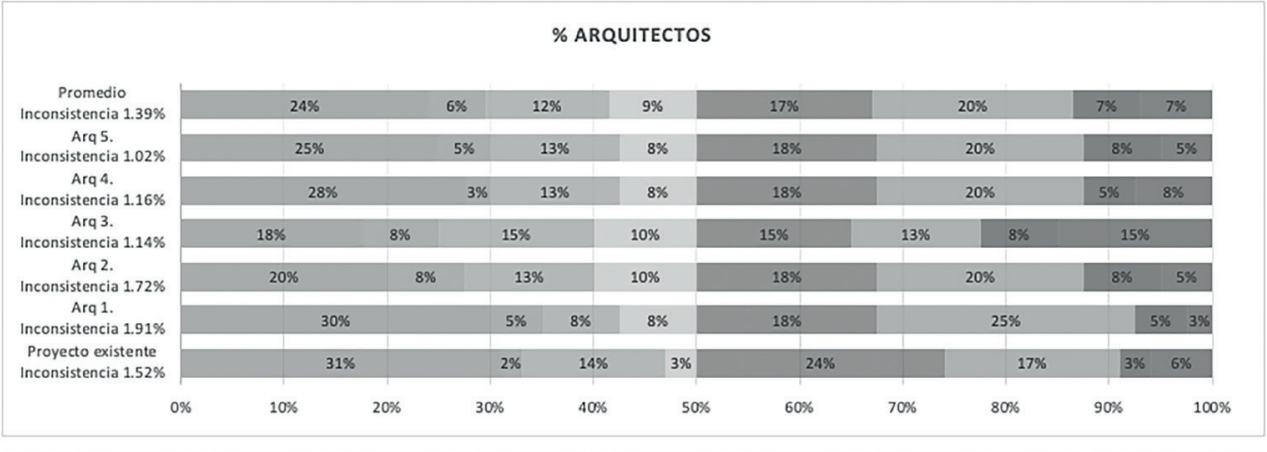

$\%$ USUARIOS

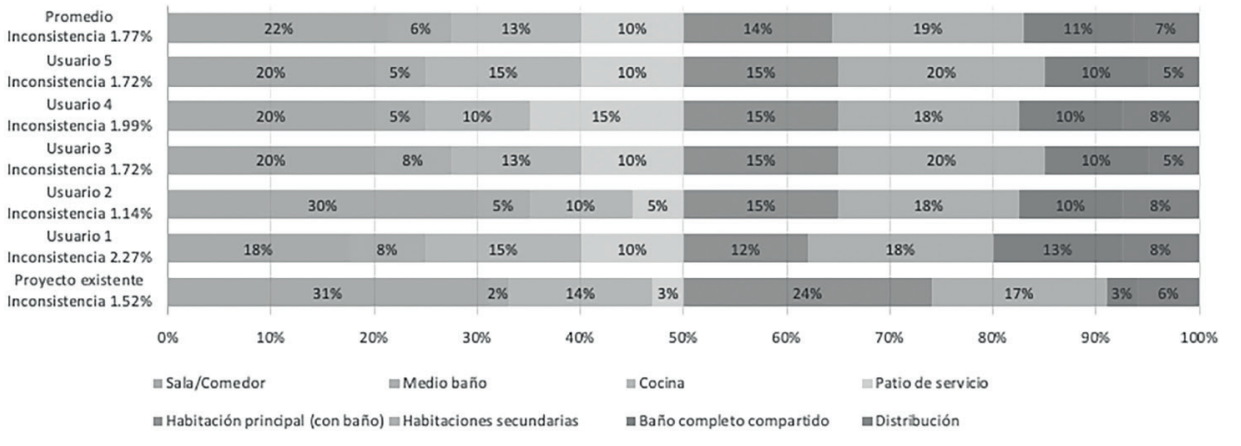

FIGURA 6.PRIORIDADES EXPRESADAS REFERENTES A LA DISTRIBUCIÓN PORCENTUAL DEL ESPACIO. FUENTE: INFORMACIÓN PROPIA Y SUPER DECLIONS.
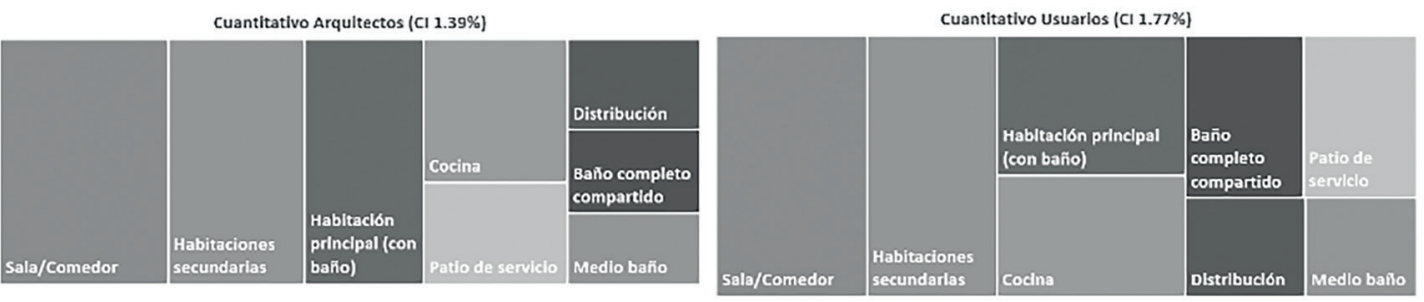

FIGURA 7.EXPRESIÓN GRÁFICA DE LAS PRIORIDADES CUALITATIVAS (PROMEDIOS) DE LOS GRUPOS ENCUESTADOS.

FUENTE: ELABORACIÓN PROPIA EMPLEANDO EL SOFTWARE EXCEL.

consideraciones, una hecha por parte de los encuestados y otra identificada:

- La consideración del uso y mobiliario deberá ser fundamental para la distribución consiente de los espacios, por ello se requiere considerar de manera directa la aplicación de conocimientos ergonómicos y no meramente utilitarios.

- Es necesario para los usuarios una explicación de la relación porcentual con las áreas destinadas (estos no cuentan con una percepción espacial de los mismos), mientras que en el caso de los arquitectos estos tuvieron grandes variaciones con respecto a la magnitud de la zona de sala/comedor (estos parecen considerar el espacio funcionalmente, pero no en magnitud de la actividad); por ello, es posible identificar que existen diferencias fundamentales de los juicios expresados por cada grupo.

Una vez identificado el comportamiento general de los encuestados en lo referente a la distribución porcentual del programa arquitectónico, puede ser considerada una distribución cuantitativa de un problema y se procederá a analizar el comportamiento de los encuestados al abordar la misma problemática desde un enfoque cualitativo.

Del análisis de las áreas y su distribución mediante un juicio comparativo de las áreas se identificaron los siguientes comportamientos:

Del análisis de importancia de espacios en un juicio pareado se identificó un incremento considerable en la inconsistencia de los juicios (figura 8); debido a que un juicio basado en aspectos cualitativos no estarán delimitados por una distribución fija o un total predeterminado, es decir, la importancia de las áreas no serán limitadas 
bajo un conocimiento consistente a priori (a menos que se tengan las herramientas para evaluar sus juicios de manera simultánea), por ello, un juicio cualitativo y su traducción a un resultado cuantitativo presentara una mayor inconsistencia.

- La inconsistencia de los juicios expresados fue mayor al CI máximo aceptable, lo cual señala que es necesario sensibilizar a los arquitectos con conceptos como lo son la correlación de importancia de áreas, actividades y espacio asignado; siendo el juicio menos inconsistente de un CI de $17.29 \%$ y uno de los más cercanos a la distribución existente; en este caso se podrá tener dos caminos de acción, uno es la mejora del juicio considerando una segunda ronda en la encuesta con una supervisión directa en caso de que no se haya comprendido la escala empleada o comprendido el impacto de los juicios expresados en la distribución, y el otro, es sensibilizar a los arquitectos con las necesidades de los usuarios y no solo la importancia supuesta de las actividades desarrolladas por los usuarios.

- La inconsistencia en el caso de los usuarios es menor que la expresada por los arquitectos al presentar un juicio con un CI de $3.88 \%$, el cual es menor al CI máximo, en este caso es importante considerar que este comportamiento es posible, a causa de que, los usuarios tienen un mayor conocimiento y sensibilidad con respecto a las características cualitativas de sus necesidades, pero sus juicios no están limitados por una escala del 100\% predefinida o limitada.

Del análisis general de los juicios emitidos en el AHP es posible identificar comportamientos específicos con respecto a la distribución de los espacios, como se muestra en la figura 10 , en los cuales es evidente el cambio de prioridades por parte de los arquitectos y usuarios, en el cual la distribución de espacios expresada por los usuarios es más cercana al proyecto existente que tiene un CI menor, aunque tampoco es lo suficientemente bajo para ser considerado como apropiado, por lo cual las distribuciones de los espacios podría ser mejorada.

Producto de estos análisis es posible identificar que los juicios expresados por los arquitectos podrían mejorar con la sensibilización respecto a aspectos cualitativos propios de las necesidades de los usuarios y a su vez los usuarios podrían mejorar la expresión de sus necesidades en áreas concretas al ser orientados en lo que respecta al funcionamiento, requerimientos ergonómicos, mobiliario y las limitantes constructivas y de funcionamiento de los espacios requeridos, cabe destacar que los resultados en cuanto a prioridades desde la perspectiva cuantitativa y cualitativa presentan diferencias notables en cuanto a consistencia y proporciones, como se muestra en la tabla 2, y de los cuales es posible identificar comportamientos específicos.

De los elementos con mayor prioridad en el caso de los arquitectos y usuarios es posible identificar que existe en su mayoría coincidencia en cuanto al espacio con mayor jerarquía cuantitativa (Sala/comedor) y una correlación menor en cuanto a otros espacios, sin embargo, en el caso de las observaciones cualitativas, los arquitectos expresaron una mayor cantidad de juicios cualitativos, donde la Cocina les representa un espacio con un gran peso jerárquico (lo cual puede estar relacionado con la concepción de su función e importancia), mientras que en el caso de los usuarios el componente con mayor jerarquía en juicios cuantitativos y cualitativos es de igual prioridad que las habitaciones principal y secundarias; quizás por concebirlas como el espacio "principal" o ser dos espacios, hecho que demuestra que los usuarios pueden expresar ciertas concepciones particulares, que podrían condicionar la distribución y espacio es del partido arquitectónico, las cuales pueden no ser evidentes para el arquitecto al partir de un pensamiento funcional o utilitario.

\section{CONCLUSIONES}

Del análisis de las maneras de desarrollar una distribución espacial de un proyecto arquitectónico, tanto de arquitectos como usuarios, fue posible identificar comportamientos particulares y oportunidades de mejorar los juicios emitidos, entre los cuales es posible identificar que existe una mayor consistencia de juicios al ser analizados con una base matemática del problema (en este caso la aproximación porcentual), sin embargo, la aproximación cualitativa nos permite matizar de mejor manera las necesidades puntuales basadas en la percepción y necesidades del usuario (las cuales pueden no se percibidas solo con una aproximación matemática del problema de jerarquía y dotación de los espacios); a su vez fue posible modelar una expresión gráfica de las suposiciones y juicios 


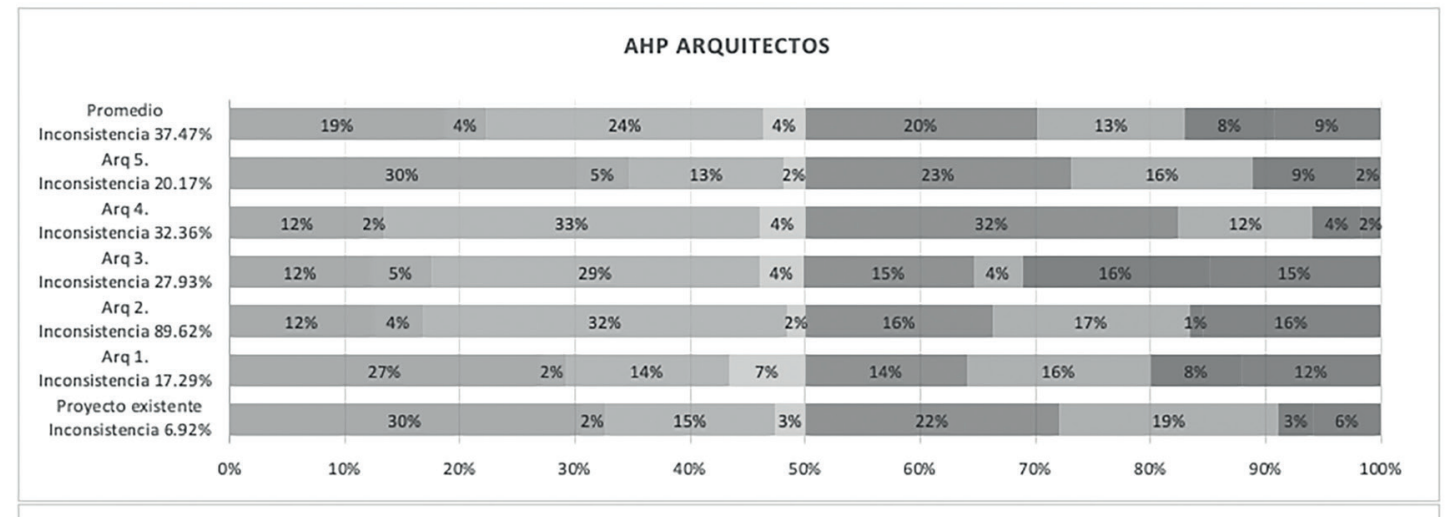

AHP USUARIOS

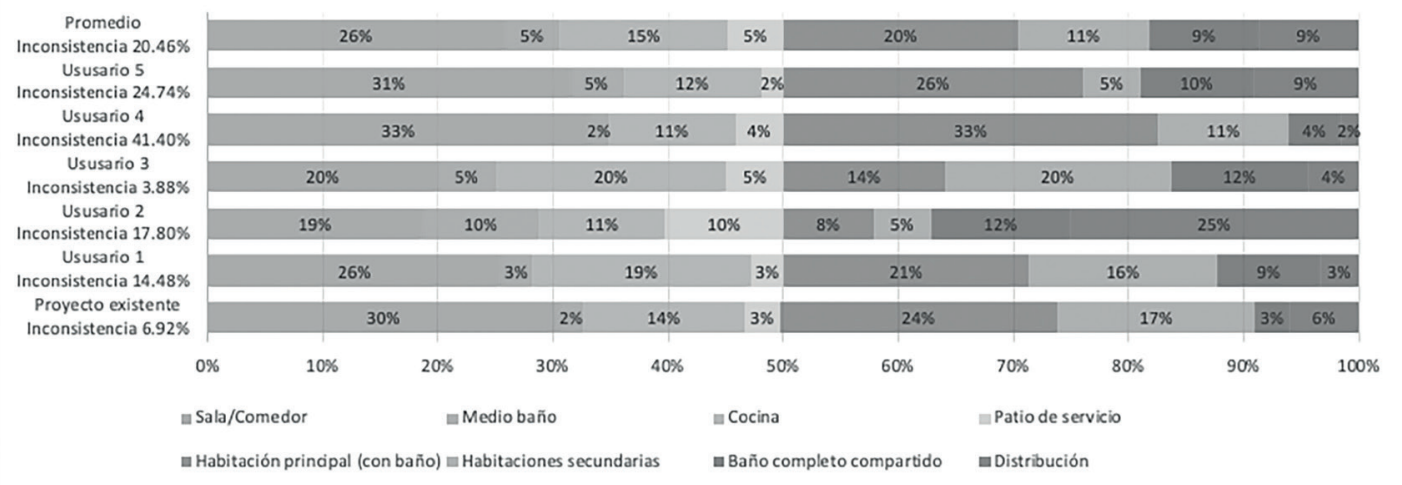

FIGURA 8.PRIORIDADES EXPRESADAS REFERENTES A LA DISTRIBUCIÓN JERÁRQUICA DEL ESPACIO. FUENTE: ELABORACIÓN PROPIA EMPLEANDO EL SOFTWARE SUPER DECISIONS.
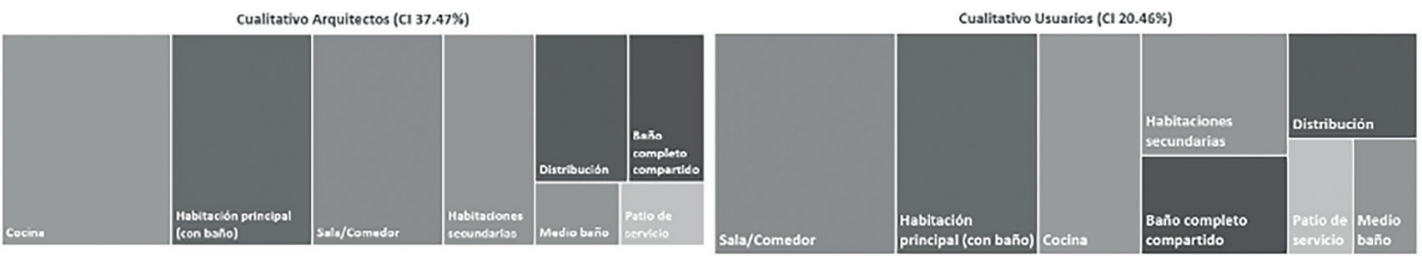

FIGURA 9. EXPRESIÓN GRÁFICA DE LOS JUICIOS PAREADOS (CUALITATIVOS) DEL PROYECTO EXISTENTE (PROMEDIO). FUENTE: ELABORACIÓN PROPIA EMPLEANDO EL SOFTWARE EXCEL.

emitidos, los cuales podrían resultar en una aproximación gráfica en magnitudes de las áreas propias del proyecto arquitectónico.

Este análisis hace hincapié en la incorporación de características cualitativas de las necesidades espaciales de los usuarios, así como la existencia de una concepción a priori que los arquitectos hacen de manera tacita al traducir necesidades 0 requerimientos a volúmenes de espacios (empleando la prioridad de cada espacio como base).

Otra aportación de la presente investigación es la sensibilización del usuario con el proceso de diseño, ya que usualmente el usuario suele concebir como un sobrecosto los servicios de un arquitecto al realizar el diseño del proyecto arquitectónico al considerar su aportación como intangible o prescindible; lo cual con los sucesos actuales con respecto a la pandemia han demostrado ser bastante graves, ya que existen muchas edificaciones de autoconstrucción, las cuales al no contar con la asesoría de un arquitecto éstas suelen no incorporar al diseño arquitectónico de las edificaciones aspectos referentes a la salud, confort y mejoras para el uso de los espacios más allá de considerarlos espacios de funciones limitadas o únicas.

Como sugerencia para estudios posteriores se recomienda la elaboración de análisis que incorporen una muestra más amplia en el análisis de los juicios, además de la incorporación de otros grupos como estudiantes o técnicos de otras áreas a fines a la elaboración de proyectos arquitectónicos, para poder así identificar otros elementos críticos que pudieran estar limitando o predisponiendo opción distributiva de las etapas analíticas del diseño. 
TABLA 2. CONCENTRADO DE PRIORIDADES CUANTITATIVAS (CT) Y CUALITATIVAS (CL) EXPRESADAS

\begin{tabular}{|c|c|c|c|c|c|c|c|c|c|c|c|c|}
\hline \multirow{3}{*}{$\begin{array}{l}\text { Programa arquitectónico } \\
\text { Sala/Comedor } \\
\end{array}$} & \multirow{2}{*}{\multicolumn{2}{|c|}{$\begin{array}{l}\text { Proyecto } \\
\text { existente }\end{array}$}} & \multicolumn{2}{|c|}{ Arq. 1} & \multicolumn{2}{|c|}{ Arq. 2} & \multicolumn{2}{|c|}{ Arq. 3} & \multicolumn{2}{|c|}{ Arq. 4} & \multicolumn{2}{|c|}{ Arq. 5} \\
\hline & & & \multirow{2}{*}{\begin{tabular}{|c|}
$\begin{array}{c}\text { Juicio } \\
\text { CT }\end{array}$ \\
$30 \%$ \\
\end{tabular}} & \multirow{2}{*}{\begin{tabular}{|c|}
$\begin{array}{c}\text { Juicio } \\
\mathrm{CL}\end{array}$ \\
$27 \%$ \\
\end{tabular}} & \multirow{2}{*}{\begin{tabular}{|c|}
$\begin{array}{c}\text { Juicio } \\
\text { CT }\end{array}$ \\
$20 \%$ \\
\end{tabular}} & \multirow{2}{*}{$\begin{array}{c}\begin{array}{c}\text { Juicio } \\
\mathrm{CL}\end{array} \\
12 \% \\
\end{array}$} & \multirow{2}{*}{$\begin{array}{c}\text { Juicio } \\
\text { CT } \\
18 \% \\
\end{array}$} & \multirow{2}{*}{$\begin{array}{c}\begin{array}{c}\text { Juicio } \\
\text { CL }\end{array} \\
12 \% \\
\end{array}$} & \multirow{2}{*}{$\begin{array}{c}\begin{array}{c}\text { Juicio } \\
\text { CT }\end{array} \\
28 \% \\
\end{array}$} & \multirow{2}{*}{$\begin{array}{c}\begin{array}{c}\text { Juicio } \\
\text { CL }\end{array} \\
12 \% \\
\end{array}$} & \multirow{2}{*}{$\begin{array}{c}\begin{array}{c}\text { Juicio } \\
\text { CT }\end{array} \\
25 \% \\
\end{array}$} & \multirow{2}{*}{$\begin{array}{c}\begin{array}{c}\text { Juicio } \\
\mathrm{CL}\end{array} \\
30 \%\end{array}$} \\
\hline & $31 \%$ & $30 \%$ & & & & & & & & & & \\
\hline Medio baño & $2 \%$ & $2 \%$ & $5 \%$ & $2 \%$ & $8 \%$ & $4 \%$ & $8 \%$ & $5 \%$ & $3 \%$ & $2 \%$ & $5 \%$ & $5 \%$ \\
\hline Cocina & $14 \%$ & $15 \%$ & $8 \%$ & $14 \%$ & $13 \%$ & $32 \%$ & $15 \%$ & $29 \%$ & $13 \%$ & $33 \%$ & $13 \%$ & $13 \%$ \\
\hline Patio de servicio & $3 \%$ & $3 \%$ & $8 \%$ & $7 \%$ & $10 \%$ & $2 \%$ & $10 \%$ & $4 \%$ & $8 \%$ & $4 \%$ & $8 \%$ & $2 \%$ \\
\hline $\begin{array}{l}\text { Habitación principal (con } \\
\text { baño) }\end{array}$ & $24 \%$ & $22 \%$ & $18 \%$ & $14 \%$ & $18 \%$ & $16 \%$ & $15 \%$ & $15 \%$ & $18 \%$ & $32 \%$ & $18 \%$ & $23 \%$ \\
\hline $\begin{array}{l}\text { Habitaciones } \\
\text { secundarias }\end{array}$ & $17 \%$ & $19 \%$ & $25 \%$ & $16 \%$ & $20 \%$ & $17 \%$ & $13 \%$ & $4 \%$ & $20 \%$ & $12 \%$ & $20 \%$ & $16 \%$ \\
\hline $\begin{array}{l}\text { Baño completo } \\
\text { compartido }\end{array}$ & $3 \%$ & $3 \%$ & $5 \%$ & $8 \%$ & $8 \%$ & $1 \%$ & $8 \%$ & $16 \%$ & $5 \%$ & $4 \%$ & $8 \%$ & $9 \%$ \\
\hline Distribución & $6 \%$ & $6 \%$ & $3 \%$ & $12 \%$ & $5 \%$ & $16 \%$ & $15 \%$ & $15 \%$ & $8 \%$ & $2 \%$ & $5 \%$ & $2 \%$ \\
\hline Total & $100 \%$ & $100 \%$ & $100 \%$ & $100 \%$ & $100 \%$ & $100 \%$ & $100 \%$ & $100 \%$ & $100 \%$ & $100 \%$ & $100 \%$ & $100 \%$ \\
\hline \multirow[b]{2}{*}{ Programa arquitectónico } & \multirow{2}{*}{\multicolumn{2}{|c|}{$\begin{array}{l}\text { Proyecto } \\
\text { existente }\end{array}$}} & \multicolumn{2}{|c|}{ Usuario 1} & \multicolumn{2}{|c|}{ Usuario 2} & \multicolumn{2}{|c|}{ Usuario 3} & \multicolumn{2}{|c|}{ Usuario 4} & \multicolumn{2}{|c|}{ Usuario 5} \\
\hline & & & $\begin{array}{c}\text { Juicio } \\
\text { CT }\end{array}$ & $\begin{array}{l}\text { Juicio } \\
\text { CL }\end{array}$ & $\begin{array}{c}\text { Juicio } \\
\text { CT }\end{array}$ & $\begin{array}{l}\text { Juicio } \\
\text { CL }\end{array}$ & $\begin{array}{l}\text { Juicio } \\
\text { CT }\end{array}$ & $\begin{array}{c}\text { Juicio } \\
\text { CL }\end{array}$ & $\begin{array}{l}\text { Juicio } \\
\text { CT }\end{array}$ & $\begin{array}{l}\text { Juicio } \\
\text { CL }\end{array}$ & $\begin{array}{l}\text { Juicio } \\
\text { CT }\end{array}$ & $\begin{array}{c}\text { Juicio } \\
\mathrm{CL}\end{array}$ \\
\hline Sala/Comedor & $31 \%$ & $30 \%$ & $18 \%$ & $26 \%$ & $30 \%$ & $19 \%$ & $20 \%$ & $20 \%$ & $20 \%$ & $33 \%$ & $20 \%$ & $31 \%$ \\
\hline Medio baño & $2 \%$ & $2 \%$ & $8 \%$ & $3 \%$ & $5 \%$ & $10 \%$ & $8 \%$ & $5 \%$ & $5 \%$ & $2 \%$ & $5 \%$ & $5 \%$ \\
\hline Cocina & $14 \%$ & $15 \%$ & $15 \%$ & $19 \%$ & $10 \%$ & $11 \%$ & $13 \%$ & $20 \%$ & $10 \%$ & $11 \%$ & $15 \%$ & $12 \%$ \\
\hline Patio de servicio & $3 \%$ & $3 \%$ & $10 \%$ & $3 \%$ & $5 \%$ & $10 \%$ & $10 \%$ & $5 \%$ & $15 \%$ & $4 \%$ & $10 \%$ & $2 \%$ \\
\hline $\begin{array}{l}\text { Habitación principal (con } \\
\text { baño) }\end{array}$ & $24 \%$ & $22 \%$ & $12 \%$ & $21 \%$ & $15 \%$ & $8 \%$ & $15 \%$ & $14 \%$ & $15 \%$ & $33 \%$ & $15 \%$ & $26 \%$ \\
\hline $\begin{array}{l}\text { Habitaciones } \\
\text { secundarias }\end{array}$ & $17 \%$ & $19 \%$ & $18 \%$ & $16 \%$ & $18 \%$ & $5 \%$ & $20 \%$ & $20 \%$ & $18 \%$ & $11 \%$ & $20 \%$ & $5 \%$ \\
\hline $\begin{array}{l}\text { Baño completo } \\
\text { compartido }\end{array}$ & $3 \%$ & $3 \%$ & $13 \%$ & $9 \%$ & $10 \%$ & $12 \%$ & $10 \%$ & $12 \%$ & $10 \%$ & $4 \%$ & $10 \%$ & $10 \%$ \\
\hline Distribución & $6 \%$ & $6 \%$ & $8 \%$ & $3 \%$ & $8 \%$ & $25 \%$ & $5 \%$ & $4 \%$ & $8 \%$ & $2 \%$ & $5 \%$ & $9 \%$ \\
\hline Total & $100 \%$ & $100 \%$ & $100 \%$ & $100 \%$ & $100 \%$ & $100 \%$ & $100 \%$ & $100 \%$ & $100 \%$ & $100 \%$ & $100 \%$ & $100 \%$ \\
\hline
\end{tabular}

FUENTE: ELABORACIÓN PROPIA CON DATOS DE ENCUESTA Y RESULTADOS DEL ANÁLLISIS DE JUICIOS EN SUPERDESICIONS.

\section{FUENTES DE CONSULTA}

Aragonés, B. P., Chaparro, G. F., Pascual, P. F. J. \& PLA, R. A. (2014),"An AHP (Analytic Hierarchy Process)/AnP (Analytic Network Process)-based multi-criteria decision approach for the selection of solar-thermal power plant investment projects", Energy, vol. 66, pp. $222-238$.

Baker, H., G. (1998), Design strategies in architecture, an approach to the analysis of form, Segunda ed., Gustavo Gili, Barcelona.

Elango, M. \& Devadas, M. (2014), "Multi-Criteria Analysis of the Design Decisions In Architectural Design Process during the PreDesign Stage", International Journal of Engineering and Technology (IJET), vol. 6, núm. 2, pp. 1033-1046.
Harputlugil, T. (2018), "Analytic Hierarchy Process (AHP) as an Assessment Approach for Architectural Design: Case Study of Architectural Design Studio", International Journal of Architecture \& Planning, vol. 6, núm. 2, pp. 217-245.

Mu, E. \& Pereyra-Rojas, M. (2017), Practical Decision An Introduction to the Analytic Hierarchy Process (AHP) Using Super Decisions v2. Pittsburgh: Springer.

Szilágyi, D. \& Bakai, N. (2019), "Use of state-of-the-art methods in the architectUral design process", Müszaki Tudományos Közlemények, vol. 11, pp. 179-182.

T. White, E. (1987), Manual de conceptos de formas arquitectónicas Cuarta reimpresión ed. Tucson, Arizona, E. U. A., Trillas. 